\title{
Combining individual animal movement and ancillary biotelemetry data to investigate population-level activity budgets
}

\author{
Brett T. McClintock, ${ }^{1,5}$ Deborah J. F. Russell, ${ }^{2,3}$ Jason Matthiopoulos, ${ }^{4}$ and Ruth King ${ }^{3}$ \\ ${ }^{1}$ National Marine Mammal Laboratory, Alaska Fisheries Science Center, NOAA National Marine Fisheries Service, \\ 7600 Sand Point Way NE, Seattle, Washington 98115 USA \\ ${ }^{2}$ Sea Mammal Research Unit, University of St Andrews, St Andrews, Fife KY16 8 LB United Kingdom \\ ${ }^{3}$ Centre for Research into Ecological and Environmental Modelling and School of Mathematics and Statistics, \\ University of St Andrews, St Andrews, Fife KY16 9LZ United Kingdom \\ ${ }^{4}$ Institute of Biodiversity Animal Health and Comparative Medicine, Graham Kerr Building, University of Glasgow, \\ Glasgow G12 8QQ United Kingdom
}

\begin{abstract}
Recent technological advances have permitted the collection of detailed animal location and ancillary biotelemetry data that facilitate inference about animal movement and associated behaviors. However, these rich sources of individual information, location, and biotelemetry data, are typically analyzed independently, with population-level inferences remaining largely post hoc. We describe a hierarchical modeling approach, which is able to integrate location and ancillary biotelemetry (e.g., physiological or accelerometer) data from many individuals. We can thus obtain robust estimates of (1) population-level movement parameters and (2) activity budgets for a set of behaviors among which animals transition as they respond to changes in their internal and external environment. Measurement error and missing data are easily accommodated using a state-space formulation of the proposed hierarchical model. Using Bayesian analysis methods, we demonstrate our modeling approach with location and dive activity data from 17 harbor seals (Phoca vitulina) in the United Kingdom. Based jointly on movement and diving activity, we identified three distinct movement behavior states: resting, foraging, and transit, and estimated population-level activity budgets to these three states. Because harbor seals are known to dive for both foraging and transit (but not usually for resting), we compared these results to a similar populationlevel analysis utilizing only location data. We found that a large proportion of time steps were mischaracterized when behavior states were inferred from horizontal trajectory alone, with $33 \%$ of time steps exhibiting a majority of dive activity assigned to the resting state. Only $1 \%$ of these time steps were assigned to resting when inferred from both trajectory and dive activity data using our integrated modeling approach. There is mounting evidence of the potential perils of inferring animal behavior based on trajectory alone, but there fortunately now exist many flexible analytical techniques for extracting more out of the increasing wealth of information afforded by recent advances in biologging technology.
\end{abstract}

Key words: animal location data; harbor seal; hierarchical model; movement model; state-space model; switching behavior; telemetry.

\section{INTRODUCTION}

Recent technological advances have permitted the collection of detailed animal location, biotelemetry, and geographic information system data (Cooke et al. 2004). The quality of location and ancillary biotelemetry information (e.g., from accelerometer, environmental, or physiological sensors) from animal-borne devices are rapidly improving with tags that are capable of storing and transmitting large amounts of data. Such technology-driven feats are exciting in themselves, but it is important to analyze these data with a view to their original objectives: understanding individual behavior

Manuscript received 8 June 2012; revised 29 October 2012; accepted 1 November 2012. Corresponding Editor: K. B. Newman.

${ }^{5}$ E-mail: brett.mcclintock@noaa.gov and predicting population redistribution. There has been a recent flood of methodological developments utilizing these data to make inferences about animal movement, space use, resource selection, and other behaviors (e.g., Morales et al. 2004, Johnson et al. 2011). However, these rich (and often interrelated) data are typically analyzed independently (e.g., Le Boeuf et al. 2000, Austin et al. 2006, Jonsen et al. 2007), with population-level inference remaining largely post hoc (e.g., Breed et al. 2009, Hanks et al. 2011).

Mechanistic multi-state movement models aim to associate different types of movement with distinct behavioral states (Blackwell 2003, Morales et al. 2004, Jonsen et al. 2005, McClintock et al. 2012). These associations are typically drawn from location data only, but inferring behavior based on trajectory alone can be difficult and problematic (Gaspar et al. 2006, 
Patterson et al. 2008). If combined in an integrated multi-state movement model, a joint analysis using both animal location and ancillary biotelemetry data could potentially improve our ability to characterize and predict behavior states (e.g., resting, feeding, migration).

Transmitters deployed on marine species often simultaneously record location (e.g., latitude, longitude, depth) and other types of biotelemetry data. Austin et al. (2006) explored grey seal foraging behavior from stomach temperature biotelemetry data, but did not use location to identify other behaviors. Higgs and Ver Hoef (2011) investigated harbor seal diving behavior from categorical time-depth recorder (TDR) data, but did not use other location data to inform their model. Johnson et al. (2011) utilized surface location and TDR data to develop a metric for foraging space use of northern fur seals, but did not utilize diving information to recreate the movement path or inform behavior states. McClintock et al. (2012) used a multi-state model to characterize grey seal movements, but only used latitude and longitude to inform movement behavior states. Clearly, because both diving and horizontal trajectory are important in distinguishing animal behaviors (e.g., resting, foraging, and transit), the prediction of behaviors and associated activity budgets (i.e., time allocations to different behavior states) would benefit by utilizing multiple sources of information.

Whether animal location, biotelemetry, or other sources of information are the basis for inference, multi-state movement model analyses have overwhelmingly involved simple examples from one (or very few) individuals (e.g., Blackwell 2003, Morales et al. 2004, Jonsen et al. 2005, McClintock et al. 2012). This is primarily because the complicated structure found in time-series of telemetry data, which often include substantial measurement error or missing observations, have necessitated an initial focus on the development of sophisticated statistical models that simultaneously accommodate the movement and observation processes. Although such analyses have been instrumental in the development and refinement of individual-based movement models, these contribute little to the overarching goal of linking animal movement and population dynamics at the interface of behavioral, population, and landscape ecology.

However, now that individual animal movement models have been developed and fitted to data, attention is beginning to shift toward utilizing these methods for population-level inferences. For example, recent seal movement studies performed separate analyses on individual (Hanks et al. 2011) or groups of individual (Breed et al. 2009) movement pathways and investigated these post hoc for population-level patterns. Jonsen et al. (2003) explored some of the advantages of "metaanalytic" methods, whereby location data from many individuals within a population may be modeled hierarchically for population-level inferences about movement and activity budgets. The advantages of hierarchical models include increased precision (i.e., greater predictive power) and the ability to "borrow" information across individuals. The latter is particularly useful for data-poor individual pathways (e.g., due to transmitter loss or failure). Despite these advantages, population-level analyses of individual movement pathways using hierarchical models are rarely performed with real data (but see Jonsen et al. 2006, Eckert et al. 2008, Langrock et al. 2012). We believe this is largely attributable to a lack of sufficient data or the computational difficulties associated with the analysis of large amounts of (imperfectly observed) location data.

Here, our objective is to extend mechanistic multistate movement models to simultaneously accommodate (1) integration of multiple sources of information about individual behavior, (2) data from multiple individuals, and (3) imperfectly observed and missing data. These extensions aim to improve our ability to identify different behaviors and investigate hypotheses about activity budgets at the population-level from a sample of individuals. After describing our statistical methodology, we provide an example using location and diving activity data from harbor seals (Phoca vitulina) in the United Kingdom.

\section{Methods}

\section{The individual model}

We are interested in combining animal location and biotelemetry data to investigate activity budgets to distinct behavior states. Similar to Morales et al. (2004), Jonsen et al. (2005), and McClintock et al. (2012), we model animal movement as a mixture of discrete-time random walks, whereby an individual may switch among a set of discrete behavior states $z=1, \ldots$, $Z$ along the movement path. Unlike previous approaches utilizing only location data to inform states, we allow each of the $Z$ behavior states to be characterized by (1) the properties of movement based on location data and (2) ancillary information from biotelemetry data.

The movement process is described by distributions for the step length and bearing of movement between consecutive positions $\left(X_{t-1}, Y_{t-1}\right)$ and $\left(X_{t}, Y_{t}\right)$ at each time step $t=1, \ldots, T$. We assume the $T$ time steps are of equal length (but see State-space formulation for imperfectly observed location data). Similar to Morales et al. (2004) and McClintock et al. (2012), we select state-dependent Weibull distributions for the step length $\left(s_{t}\right)$ and wrapped Cauchy distributions for the bearing $\left(\phi_{t}\right)$ of movement:

$$
f\left(s_{t} \mid \boldsymbol{a}, \boldsymbol{b}, z_{t}=i\right)=\frac{b_{i}}{a_{i}}\left(\frac{s_{t}}{a_{i}}\right)^{b_{i}-1} \exp \left[-\left(s_{t} / a_{i}\right)^{b_{i}}\right]
$$

and

$$
f\left(\phi_{t} \mid \boldsymbol{\rho}, z_{t}=i\right)=\frac{1}{2 \pi}\left(\frac{1-\rho_{i}^{2}}{1+\rho_{i}^{2}-2 \rho_{i} \cos \left(\phi_{t}-\phi_{t-1}\right)}\right)
$$


for scale parameter $a_{z}>0$, shape parameter $b_{z}>0$, directional persistence parameter $-1<\rho_{z}<1$, and $0<$ $\phi_{t}<2 \pi$. For switches between behavior states, we assign a first-order Markov categorical distribution, $\left[z_{t} \mid \psi, z_{t-1}\right.$ $=k] \sim$ Categorical $\left(\psi_{k, 1}, \ldots, \psi_{k, Z}\right)$, for $k=1, \ldots, Z$ where $\psi_{k, i}$ is the probability of switching from state $k$ at time $t-1$ to state $i$ at time $t$, and $\sum_{j=1}^{Z} \psi_{k, j}=1$.

Although movement behavior state could be assigned solely based on these movement characteristics (e.g., Morales et al. 2004, McClintock et al. 2012), we wish to incorporate additional information about behavior states provided by ancillary biotelemetry data. Assuming independence between step length and bearing, we therefore incorporate biotelemetry data $\left(\omega_{t}\right)$ for each time step into a joint conditional likelihood:

$$
\begin{aligned}
& f(\boldsymbol{s}, \boldsymbol{\phi}, \boldsymbol{\omega}, \mathbf{z} \mid \boldsymbol{\theta}) \\
& \quad=\prod_{t=1}^{T} f\left(\boldsymbol{s}_{t} \mid \boldsymbol{\theta}, z_{t}\right) f\left(\phi_{t} \mid \boldsymbol{\theta}, z_{t}\right) f\left(\omega_{t} \mid \boldsymbol{\theta}, z_{t}\right) f\left(z_{t} \mid \boldsymbol{\theta}, z_{t-1}\right)
\end{aligned}
$$

where $\boldsymbol{\theta}$ denotes the set of all model parameters. The exact distribution for $f\left(\omega_{t} \mid \boldsymbol{\theta}, z_{t}\right)$ will depend on the ancillary biotelemetry data available. We also note that in some situations it may be more natural to condition latent states on the ancillary data (see Discussion).

For illustration, suppose that along with spatial locations for a marine mammal central-place forager, diving activity data are also available (e.g., from pressure sensors) for each time step. Suppose $Z=3$ movement behavior states are of interest: resting (state $\mathrm{R}$ ), foraging (state F), and transit (state T). Each of these states could be characterized by different distributions for step length (e.g., $a_{\mathrm{R}}<a_{\mathrm{F}}<a_{\mathrm{T}}$ ) and directional persistence (e.g., $\rho_{\mathrm{F}}<\rho_{\mathrm{T}}$ ). These states could also be characterized according to diving activity, such as the percent of each time step spent in various depth categories. For this species, suppose the resting state is often associated with surface time or shallow dives, the transit state is often associated with moderate dives, and the foraging state is often associated with deep dives (see Fig. 1a). One could specify $\left[\omega_{t} \mid \tau, z_{t}=i\right]$ $\sim \operatorname{Dirichlet}\left(\tau_{\mathrm{s}, i}, \tau_{\mathrm{m}, i}, \tau_{\mathrm{d}, i}\right)$, where $\boldsymbol{\omega}_{t}=\left(\omega_{\mathrm{s}, t}, \omega_{\mathrm{m}, t}, \omega_{\mathrm{d}, t}\right)$ are the respective percentages of time step $t$ spent in shallow, moderate, and deep dives $\left(\sum \boldsymbol{\omega}_{t}=1\right)$. The statedependent concentration vector $\tau_{,, z}$ could be constrained according to prior beliefs about diving behavior. For example, one may believe the energetic costs of deep dives are prohibitive except when foraging, such that $\tau_{\mathrm{s}, \mathrm{R}}>\tau_{\mathrm{m}, \mathrm{R}}>\tau_{\mathrm{d}, \mathrm{R}}, \tau_{\mathrm{d}, \mathrm{F}}>\tau_{\mathrm{m}, \mathrm{F}}>$ $\tau_{\mathrm{s}, \mathrm{F}}$, and $\tau_{\mathrm{m}, \mathrm{T}}>\tau_{\mathrm{s}, \mathrm{T}}>\tau_{\mathrm{d}, \mathrm{T}}$.

As another example, suppose that along with spatial locations for an endothermic marine predator, stomach temperature biotelemetry data (sensu Austin et al. 2006) are also available for each time step. Further, suppose $Z=$ 3 behavior states are of interest: resting (state R), feeding (state D), and searching (state S). As before, each of these states could be characterized by different distributions for step length and directional persistence, but because marine prey will tend to be of lower body temperature than their endothermic predators, these states could also be partially characterized according to stomach temperature (e.g., the minimum or average stomach temperature for each time step). For example, the resting and searching states would most often be associated with no feeding (i.e., higher stomach temperatures), and the foraging state would most often be associated with feeding (i.e., lower stomach temperatures). A simple approach would be to assume $\left[\omega_{t} \mid \boldsymbol{\mu}, \boldsymbol{\sigma}, z_{t}=i\right] \sim \operatorname{lognormal}\left(\mu_{i}, \sigma_{i}^{2}\right)$, where $\omega_{t}$ is the stomach temperature for time step $t$ (Fig. 1b). Here, $\mu_{z}$ and $\sigma_{z}^{2}$ are (state-dependent) location and scale parameters, respectively, such that the expected value $E\left(\omega_{t} \mid z_{t}=i\right)$ $=\exp \left(\mu_{i}+\sigma_{i}^{2} / 2\right)$. To reflect the expected relationship between stomach temperature and feeding, one could enforce constraints such as $\mu_{i}>\mu_{\mathrm{D}}$ or $\exp \left(\mu_{i}+\sigma_{i}^{2} / 2\right)>$ $\exp \left(\mu_{\mathrm{D}}+\sigma_{\mathrm{D}}^{2} / 2\right)$ for $i \in\{\mathrm{R}, \mathrm{S}\}$.

Based on the joint likelihood for all sources of information, the predicted state assignments for each time step can be used to examine activity budgets at various temporal scales (Morales et al. 2004, McClintock et al. 2012). Depending on time step lengths, different hypotheses about behavioral patterns (e.g., daily, monthly, or seasonal patterns) may be investigated. By extending this individual model using a hierarchical framework, population-level hypotheses about activity budgets can be readily addressed (e.g., sex- or age-dependent time allocations to different states).

\section{The hierarchical population-level model}

Extending the individual model to a hierarchical population-level model is straightforward in theory. Similar to the individual model (Eq. 1), the movement process is described by the step length $\left(s_{n, t}\right)$ and bearing $\left(\phi_{n, t}\right)$ of movement between consecutive positions $\left(X_{n, t-1}\right.$, $\left.Y_{n, t-1}\right)$ and $\left(X_{n, t}, Y_{n, t}\right)$ at each time step $t=1, \ldots, T_{n}$ for individual $n=1, \ldots, N$. We again assume the $T_{n}$ time steps are of equal length (but see State-space formulation for imperfectly observed location data). For each time step, there are ancillary biotelemetry data $\left(\omega_{n, t}\right)$ that inform behavioral state $\left(z_{n, t}\right)$. We now assume $\left[s_{n, t} \mid \boldsymbol{a}_{n}, \boldsymbol{b}_{n}, z_{n, t}=i\right]$ $\sim$ Weibull $\left(a_{n, i}, b_{n, i}\right)$ for state-specific scale parameter $a_{n, i}$ $>0$ and shape parameter $b_{n, i}>0$, where $\log \left(a_{n, z}\right) \sim \mathcal{N}\left(\alpha_{z}\right.$, $\left.\sigma_{\alpha_{z}}^{2}\right)$ and $\log \left(b_{n, z}\right) \sim \mathcal{N}\left(\beta_{z}, \sigma_{\beta_{z}}^{2}\right)$, and $\left[\phi_{n, t} \mid \boldsymbol{\rho}_{n}, z_{n, t}=i\right] \sim$ wCauchy $\left(\phi_{n, t-1}, \rho_{n, i}\right)$ with bearing $0 \leq \phi_{n, t}<2 \pi$ and state-specific directional persistence $-1<\rho_{n, z}<1$, where $\tanh ^{-1}\left(\rho_{n, z}\right) \sim \mathcal{N}\left(r_{z}, \sigma_{r_{z}}^{2}\right)$. The biotelemetry data have probability density function $f\left(\omega_{n . t} \mid \boldsymbol{\theta}, z_{n, t}\right)$, and depending on the nature of these data, this distribution may also be modeled hierarchically. Assuming independence, we extend Eq. 1 to $N$ individuals via the joint populationlevel conditional likelihood:

$$
\begin{aligned}
f(\boldsymbol{s}, \boldsymbol{\phi}, \boldsymbol{\omega}, \mathbf{z} \mid \boldsymbol{\theta})= & \prod_{n=1}^{N} \prod_{t=1}^{T_{n}}\left[f\left(s_{n, t} \mid \boldsymbol{\theta}, z_{n, t}\right) f\left(\phi_{n, t} \mid \boldsymbol{\theta}, z_{n, t}\right)\right. \\
& \left.\times f\left(\omega_{n, t} \mid \boldsymbol{\theta}, z_{n, t}\right) f\left(z_{n, t} \mid \boldsymbol{\theta}, z_{n, t-1}\right)\right] .
\end{aligned}
$$




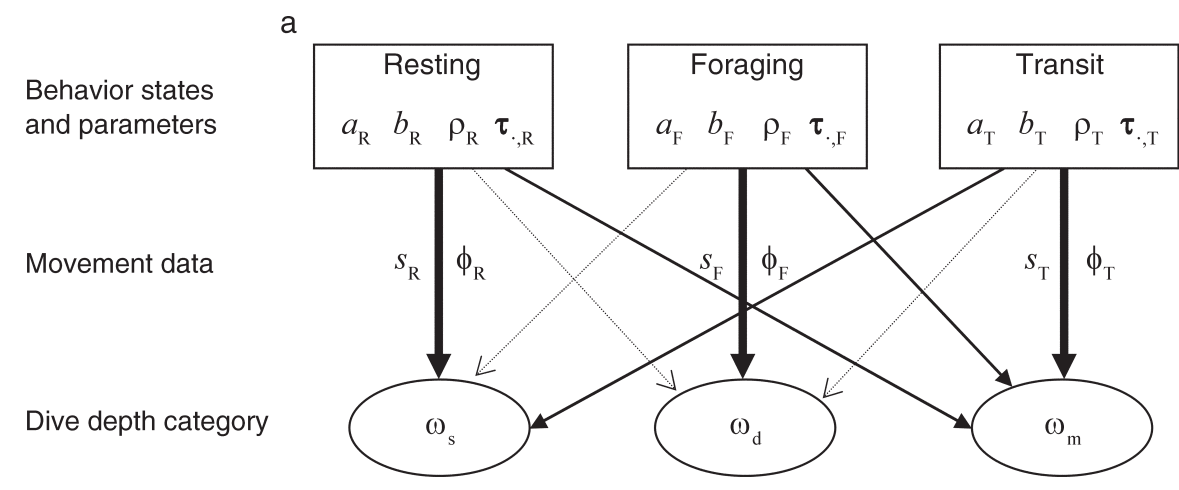

b

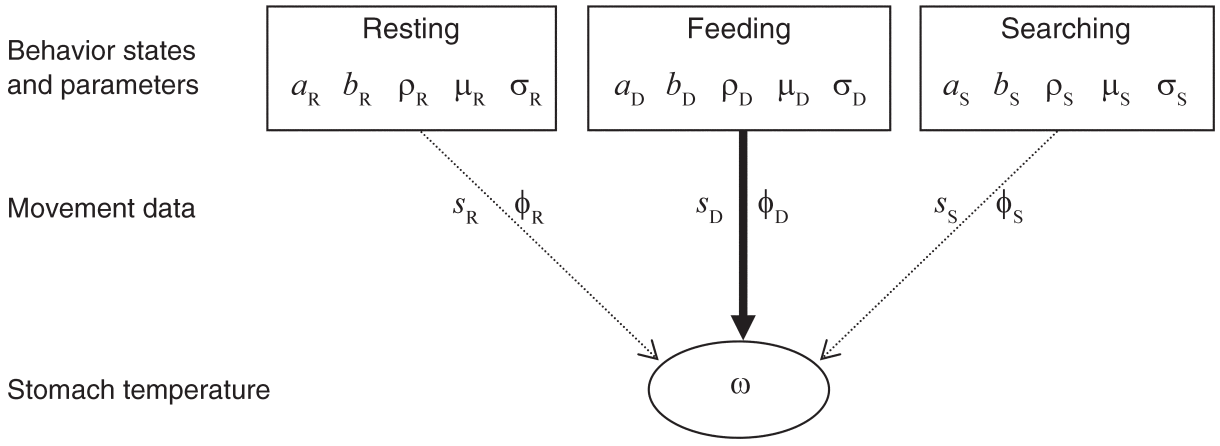

FIG. 1. Example characterizations of three latent behavior states based on both movement and ancillary biotelemetry data. Behavior state-dependent movement parameters are step length scale $a$, step length shape $b$, and directional persistence $\rho$. In panel (a), the behavior states (resting [R], foraging [F], and transit [T]) for a marine mammal central-place forager are characterized by movement (step length $[s]$ and bearing $[\phi])$ and the proportion of time spent in three dive depth categories $\left(\omega_{\mathrm{s}}\right.$, shallow; $\omega_{\mathrm{m}}$, moderate; and $\omega_{\mathrm{d}}$, deep), where shallow diving is most strongly associated with resting, moderate dives are most strongly associated with transit, and deep dives are most strongly associated with foraging. The Dirichlet model parameters $\tau_{\cdot z}=\left(\tau_{\mathrm{s}, z}, \tau_{\mathrm{m}, z}, \tau_{\mathrm{d}, z}\right)$ reflect these expected relationships between dive depth category and behavior state. For each behavior state, arrow widths are proportional to the expected time spent in each dive depth category. In panel (b), the behavior states (resting, feeding [D], and searching $[\mathrm{S}])$ for an endothermic marine predator are characterized by movement and stomach temperature $(\omega)$, where arrow width is proportional to the probability of a lower stomach temperature. The lognormal model parameters $\left(\mu_{z}\right.$ and $\left.\sigma_{z}^{2}\right)$ reflect these expected relationships between stomach temperature and the behavior state.

\section{State-space formulation for imperfectly observed location data}

Animal location and biotelemetry data are often imperfectly observed, with location measurement error, temporal irregularity, and missing observations. Environmental conditions may affect the timing and location of fixes, as may animal behavior (Frair et al. 2010). For reliable inference, these complications must be accounted for when applying the mechanistic movement models described in The individual model and The hierarchical population-level model. To account for spatial error and temporal irregularity, we adopt an observation model to accompany our discrete-time movement process model in a state-space formulation (Jonsen et al. 2005, McClintock et al. 2012). By simultaneously modeling the observation and movement processes, we are able to predict the movement path for each individual at regular time steps while accounting for location error and missing observations.
Under imperfect observation, the location data consist of the observed locations $\left(x_{n, t, i}, y_{n, t, i}\right)$ for individual $n=1, \ldots, N$, time step $t=1, \ldots, T_{n}$, and observation $i=1, \ldots, k_{n, t}$ (where time steps with $k_{n, t}=0$ have no observed locations). Similar to Jonsen et al. (2005) and McClintock et al. (2012), we assume that individuals travel in a straight line between times $t-1$ and $t$. The observed locations $\left(x_{n, t, i}, y_{n, t, i}\right)$ are then related to the temporally regular and true locations $\left(X_{n, t}, Y_{n, t}\right)$ via

$$
\begin{aligned}
& x_{n, t, i}=\left(1-j_{n, t, i}\right) X_{n, t-1}+j_{n, t, i} X_{n, t}+\varepsilon_{x_{n, t, i}} \\
& y_{n, t, i}=\left(1-j_{n, t, i}\right) Y_{n, t-1}+j_{n, t, i} Y_{n, t}+\varepsilon_{y_{n, t, i}}
\end{aligned}
$$

with error terms $\varepsilon_{x_{n, t, i}} \sim \mathcal{N}\left(0, \sigma_{x}^{2}\right)$ and $\varepsilon_{y_{n, t, i}} \sim \mathcal{N}\left(0, \sigma_{y}^{2}\right)$, where $j_{n, t, i} \in(0,1]$ is the proportion of the time interval between locations $\left(X_{n, t-1}, Y_{n, t-1}\right)$ and $\left(X_{n, t}, Y_{n, t}\right)$ at which the $i$ th observation between times $t-1$ and $t$ was obtained. Time intervals with no observations (i.e., $k_{n, t}=$ 0) do not contribute to the observation model likeli- 


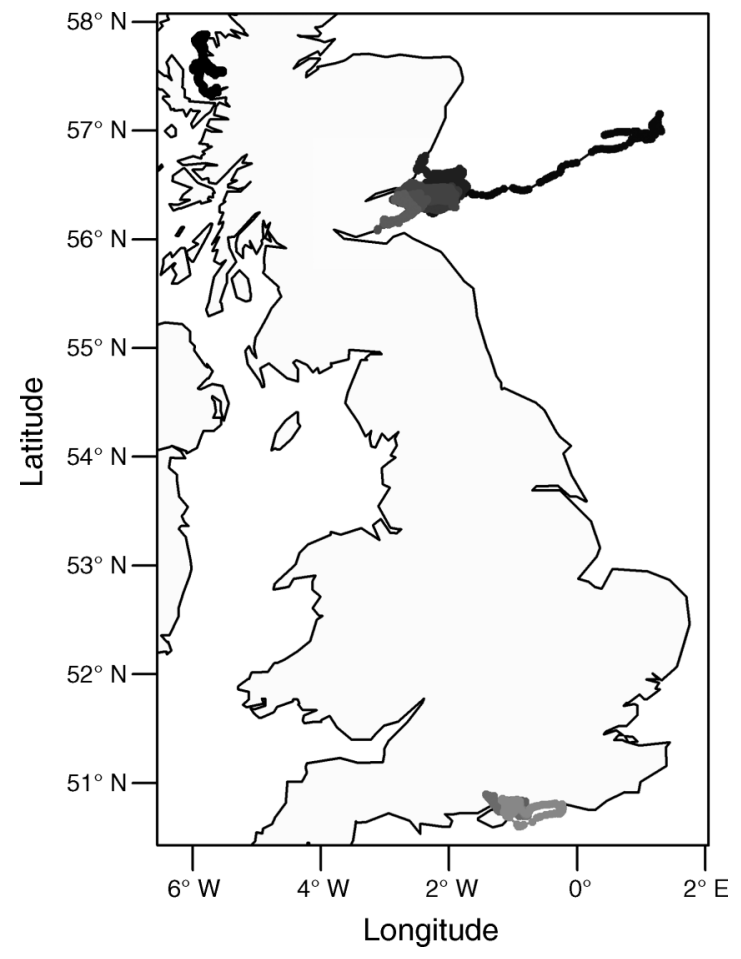

FIG. 2. Observed locations for 17 harbor seals residing in the United Kingdom.

hood. The state-space model conditional likelihood for the observation and movement processes is therefore

$$
\begin{aligned}
f(\boldsymbol{x}, \boldsymbol{y}, \boldsymbol{s}, \boldsymbol{\phi}, \boldsymbol{\omega}, \mathbf{z} \mid \boldsymbol{\theta}, \boldsymbol{z}) \\
=\prod_{n=1}^{N} \prod_{t=1}^{T_{n}} f\left(s_{n, t} \mid \boldsymbol{\theta}, z_{n, t}\right) f\left(\phi_{n, t} \mid \boldsymbol{\theta}, z_{n, t}\right) f\left(\omega_{n, t} \mid \boldsymbol{\theta}, z_{n, t}\right) \\
\quad \times f\left(z_{n, t} \mid \boldsymbol{\theta}, z_{n, t-1}\right) \prod_{i=1}^{k_{n, t}} f\left(x_{n, t, i}, y_{n, t, i} \mid \boldsymbol{\theta}\right)
\end{aligned}
$$

where $\boldsymbol{\theta}$ again denotes the set of all model parameters (including $\boldsymbol{X}$ and $\boldsymbol{Y}$ ).

\section{Example: Harbor Seals in the United Kingdom \\ Example background and methods}

As central-place foragers typically traveling between haul-out and foraging areas, harbor seals exhibit distinctive movement behaviors that fit naturally within our proposed framework. We therefore demonstrate the use of our model on location and dive data from $N=17$ adult harbor seals (10 males and 7 females) that haul out in the United Kingdom (Fig. 2). The data were obtained from GPS phone tags (Sea Mammal Research Unit [SMRU], St Andrews, United Kingdom) between 2 May and 20 July 2008 (four individuals), 19 March and 5 August 2009 (five individuals), and 20 February and 10 July 2011 (eight individuals). The tags were attached to animals' fur with adhesive, and animals were caught and processed using the techniques described in Sharples et al. (2012) under the terms of licenses issued by the U.K. Home Office under the Animals (Scientific Procedures) Act 1986. Locations were transmitted opportunistically, but dive data were continuously recorded and binned according to the amount of time spent at least $1.5 \mathrm{~m}$ below the surface in 2-h periods. The transmitted biotelemetry data $\left(\omega_{n, t}\right)$ therefore consisted of the proportion of each 120 -minute time step spent diving below $1.5 \mathrm{~m}$.

Locations were attempted at least every 30 minutes, but because positions are only attainable when an individual surfaces, locations were obtained at irregular time intervals. For about half of the individuals, following any "dry" period where a transmitter remained out of water for more than 10 minutes, no new fixes were attempted until the transmitter returned to water continuously for 40 seconds. In other words, there were frequent missing data when an individual was either hauled out or underwater. To account for measurement error and temporal irregularity of observations, we therefore used the state-space formulation described above to predict the movement path for each individual at regular time steps.

The observed location data consisted of 76511 temporally irregular locations $\left(x_{n, t, i}, y_{n, t, i}\right)$ for $n=$ $1, \ldots, 17, t=1, \ldots, T_{n}$, and $i=1, \ldots, k_{n, t}$ (where time steps with $k_{n, t}=0$ have no observed location). All observed locations were those retained after the data had been filtered using the SMRU protocol for cleaning telemetry data (Russell et al. 2011). Based on the scale of movements of harbor seals (Cunningham et al. 2009) and the frequency of observations, we specified regular time steps of 120 minutes between times $t-1$ and time $t$ for $t=1, \ldots, T_{n}\left(\sum_{n=1}^{N} T_{n}=17\right.$ 761). Based on 120minute intervals, individual seal pathways ranged from $\min \left(T_{n}\right)=121$ to $\max \left(T_{n}\right)=1789$ time steps. Our selection of 120-minute intervals reflects a trade-off between computational efficiency, the temporal resolution of the data, and an acceptable temporal resolution for inference about harbor seal movement behavior. For each seal, the first 120-minute interval began after deployment, and the last interval ended after the final observation.

Previous studies of pinniped movement first removed apparent haul-out or resting periods from individual pathways prior to making inferences about foraging and transitory movements based on horizontal trajectory alone (Breed et al. 2009, Hanks et al. 2011). We are interested in characterizing and predicting movement behaviors associated with resting, foraging, and transit for entire individual pathways using both horizontal trajectory and dive activity data. We also wish to investigate population-level differences in activity budgets and movement characteristics between males and females.

We define foraging movements as those characteristic of area-restricted searches. We define transit movements as predominantly travelling among haul-out and forag- 


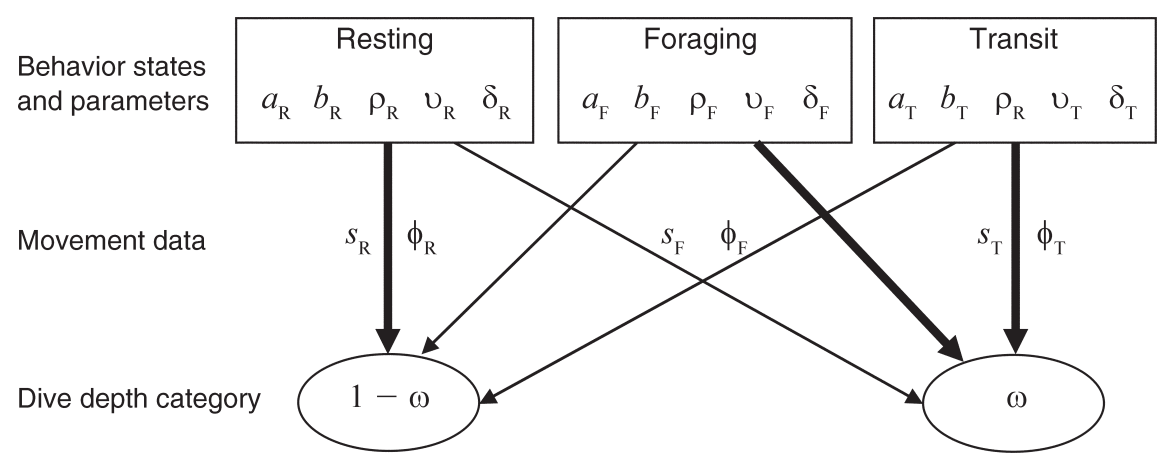

FIG. 3. Characterization of three latent behavior states, resting (R), foraging (F), and transit (T), for U.K. harbor seals based on movement direction $(\phi)$, step length $(s)$, and dive activity data. The dive activity data consist of the proportion of each time step spent $1.5 \mathrm{~m}$ below the surface, where dives below $1.5 \mathrm{~m}$ are more strongly associated with foraging and transit. For each behavior state, arrow widths are proportional to the expected time spent in each dive depth category $(\omega=$ diving, $1-\omega=$ not diving). The Beta model parameters $\left(v_{z}\right.$ and $\left.\delta_{z}\right)$ reflect these expected relationships between dive depth category and behavior state.

ing areas, although we recognize that seals may opportunistically feed while travelling. We define resting movements as those with limited horizontal displacement. As well as resting on land, known as hauling out, seals also rest on the surface at sea presumably to facilitate food processing (Sparling et al. 2007). In terms of trajectory, we would expect speeds to be low during resting and low-to-moderate during foraging, with little directional persistence. During transit, we would expect higher speeds and greater directional persistence. Within a Bayesian analysis paradigm, we assigned priors for the movement process parameters to reflect these expectations (see Appendix A for prior specifications and prior sensitivity analysis results).

It can be difficult to distinguish resting and foraging solely from horizontal displacement (particularly when individuals are resting at sea or in the vicinity of haulout sites). Because harbor seals dive for both the purposes of travelling and foraging, we will utilize both horizontal trajectory and the proportion of each time step spent below $1.5 \mathrm{~m}$ to help identify the three states (Fig. 3). Independent of bearing and step length, we assume a priori that diving below $1.5 \mathrm{~m}$ is equally likely to be associated with the foraging or transit states. We therefore assume $\left[\omega_{n, t} \mid \mathbf{v}, \boldsymbol{\delta}, z_{n, t}=i\right] \sim \operatorname{Beta}\left(\mathrm{v}_{i, j}, \delta_{i, j}\right)$ for $i$ $=\mathrm{R}, \mathrm{F}, \mathrm{T}$, where $v_{\mathrm{F}, j}=v_{\mathrm{T}, j}, \delta_{\mathrm{F}, j}=\delta_{\mathrm{T}, j}$, and $j=1$ if individual $n$ is male ( $j=2$ if female). We assigned priors based on the expected relationships between time spent diving below $1.5 \mathrm{~m}$ and the three movement behavior states (Appendix A).

For switches between movement behavior states, we assigned a first-order Markov categorical distribution, $\left[z_{n, t} \mid \psi, \mathrm{z}_{n, t-1}=k\right] \sim \operatorname{Categorical}\left(\psi_{k, \mathrm{R}, j}, \psi_{k, \mathrm{~F}, j}, \psi_{k, \mathrm{~T}, j}\right)$, for $k=\mathrm{R}, \mathrm{F}, \mathrm{T}$ and $j=1,2$, where $\psi_{k, i, j}$ is the probability of switching from state $k$ at time $t-1$ to state $i$ at time $t$ for sex $j$. This sex-dependent state-switching model allows differences between male and female state transition probabilities to be investigated.

We fit the complete state-space model using a Markov chain Monte Carlo (MCMC) algorithm written in the $\mathrm{C}$ programming language (see Supplement). When posterior conditional distributions were not of a standard form, random walk Metropolis-Hastings parameter updates were used (see Appendix B). Any missing location or dive data were imputed as latent variables within the MCMC algorithm. After initial pilot tuning and burn-in of 50000 iterations, a single chain of 500000 iterations was attained. To reduce memory requirements, we thinned the chain by 250 iterations for calculating posterior summaries. CPU time was approximately 72 hours on a machine running 64-bit Windows $7(3.4 \mathrm{GHz}$ Intel Core i7 processor, 16Gb RAM; Microsoft, Redmond, Washington, USA).

To examine whether the inclusion of ancillary diving data improve our ability to identify and characterize the three movement behavior states, we performed an additional harbor seal analysis based solely on the location data. Except for excluding $f\left(\omega_{n, t} \mid \boldsymbol{v}, \boldsymbol{\delta}, z_{n, t}\right)$ from the state-space model likelihood, the model specification and MCMC algorithm were identical.

\section{Example results and discussion}

Fig. 4 illustrates the predicted movement path and behavioral state assignments for two harbor seals. Based on estimated population-level activity budgets, both males and females devoted the majority of 120-minute time steps to foraging activity (Table 1). Males devoted a greater portion of the study period to the resting and transit states than females, and females devoted a greater portion to the foraging state than males. Individual females exhibited activity budgets ranging from 0.20 to 0.40 for resting, 0.54 to 0.74 for foraging, and 0.02 to 0.12 for transit. Individual males exhibited greater variability in activity budgets, ranging from 0.28 to 0.68 for resting, 0.25 to 0.61 for foraging, and 0.03 to 0.34 for transit. Based on posterior summary statistics for $r_{z, j}, \alpha_{z, j}$, and $\beta_{z, j}$, no significant differences in statespecific movement characteristics were found between males and females. In addition, the posterior distributions for $\sigma_{r_{z, j}}^{2}, \sigma_{\alpha_{2, j}}^{2}$, and $\sigma_{\beta_{z, j}}^{2}$ (the individual-level 


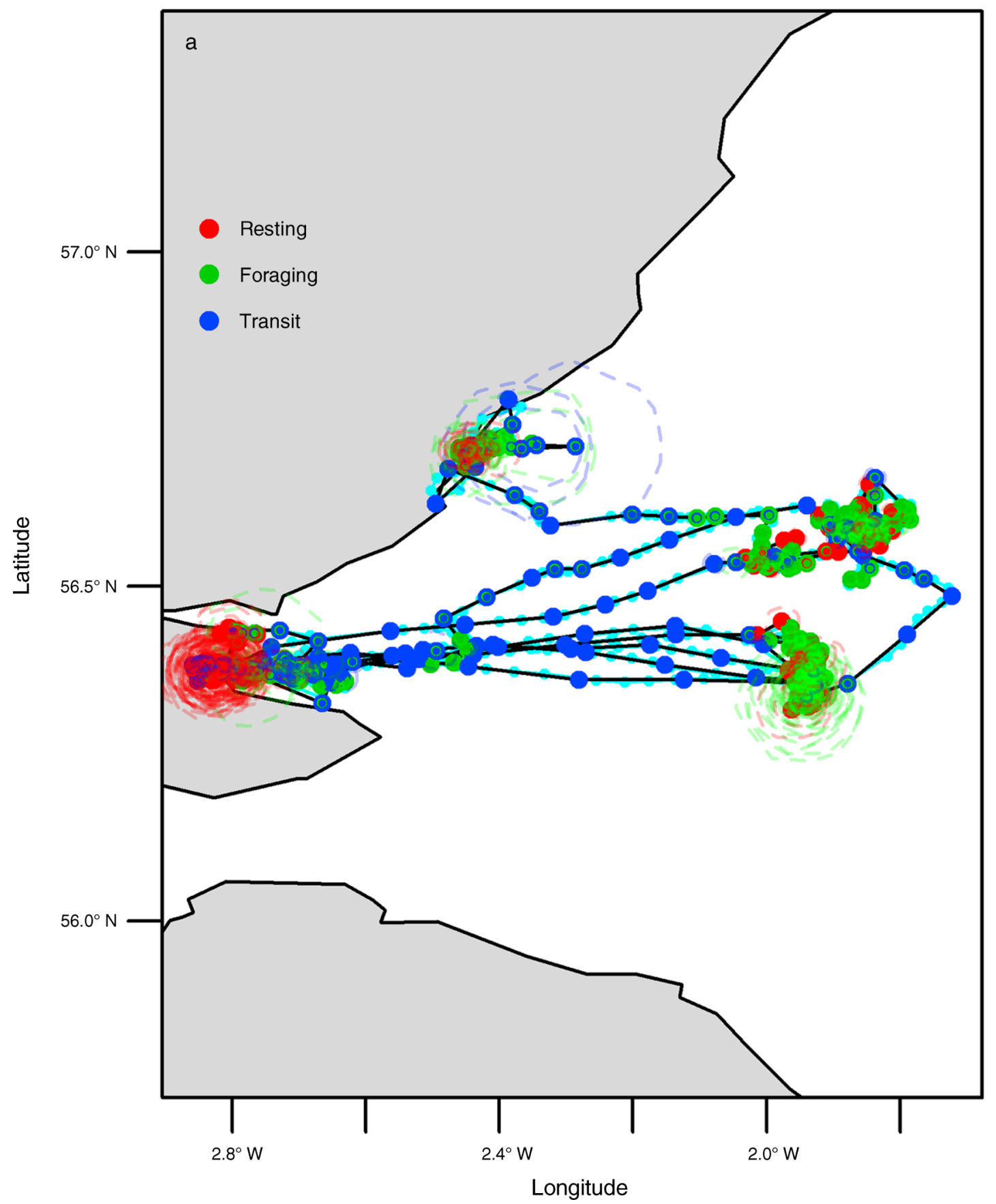

FIG. 4. Predicted locations and movement behavior states for two harbor seals in the United Kingdom: (a) a male in southeastern Scotland and (b; on following page) a female in northwestern Scotland. Estimated movement states for the predicted locations correspond to resting (red), foraging (green), and transit (blue) movement behavior states. Light blue points indicate observed locations. Uncertainty in the state assignments ( $<95 \%$ posterior probability) is indicated by hollow circles within predicted locations. Uncertainty in predicted locations is indicated by $95 \%$ credible bands (dashed lines).

variation) were similar between the sexes (see Appendix C for detailed posterior summaries).

Compared to transitory movements, resting and foraging movements exhibited significantly shorter step lengths and less directional persistence for both sexes (see Appendix C). The posterior bivariate densities for step length $\left(s_{n, t}\right)$ and the proportion of time spent diving below $1.5 \mathrm{~m}\left(\omega_{n, t}\right)$ were similar for males and females (Fig. 5), but some differences were found with respect to state transition probabilities. For example, males were more likely to remain in the resting state (posterior mean for $\psi_{R, R, 1}=0.80 ; 95 \%$ highest posterior density 


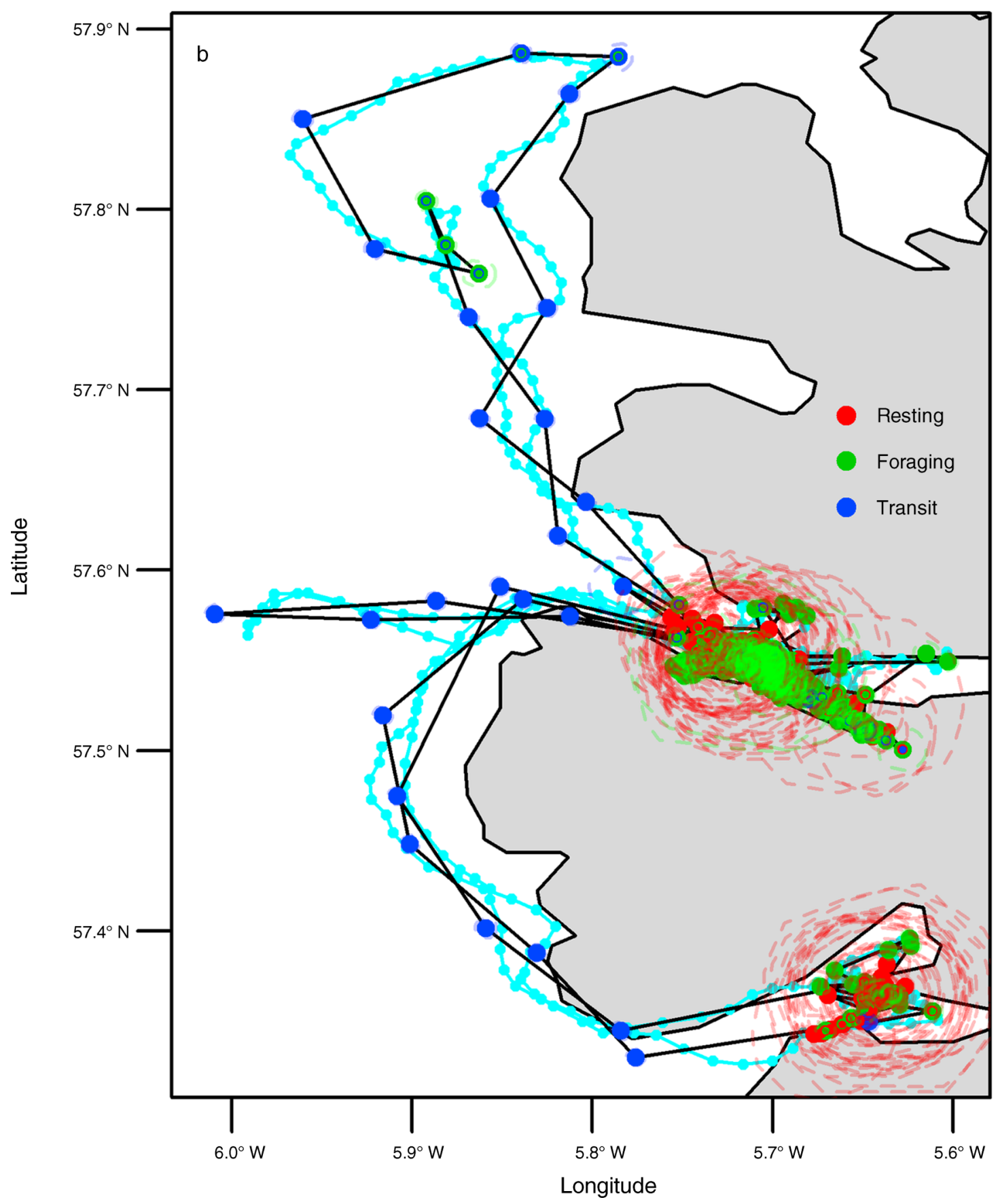

FIG. 4. Continued.

interval [HPDI], 0.79-0.81) than females $\left(\psi_{\mathrm{R}, \mathrm{R}, 2}=0.71\right.$; 95\% HPDI, 0.69-0.73), and females were slightly more likely to remain in the foraging state $\left(\psi_{\mathrm{F}, \mathrm{F}, 2}=0.85 ; 95 \%\right.$ HPDI, 0.84-0.86) than males ( $\psi_{\mathrm{F}, \mathrm{F}, 1}=0.81 ; 95 \%$ HPDI, 0.80-0.82). Sharples et al. (2012) found that males travelled farther than females, and this may explain why males spent a greater proportion of time in the transiting state and females were more likely to stay in the foraging state.
We expected to see some variability in activity budgets and movement characteristics because the study period overlapped with the breeding season each year, which typically begins in early June. We did not find any significant differences pre- and post-breeding season with respect to movement characteristics or time spent diving below $1.5 \mathrm{~m}$. However, we found small differences in time allocations to the different states, with females spending slightly more time in the transit state 
TABLE 1. Estimated proportion of 120 -minute time steps assigned to $Z=3$ movement behavior states (resting, foraging, and transit) for $N=17$ (10 male, 7 female) harbor seals in the United Kingdom.

\begin{tabular}{|c|c|c|c|c|c|c|c|}
\hline \multirow[b]{3}{*}{ Sex } & \multirow[b]{3}{*}{ Behavior state } & \multicolumn{3}{|c|}{ Dive and location data } & \multicolumn{3}{|c|}{ Location data only } \\
\hline & & \multirow[b]{2}{*}{ Time allocation } & \multicolumn{2}{|c|}{$95 \%$ HPDI } & \multirow[b]{2}{*}{ Time allocation } & \multicolumn{2}{|c|}{$95 \%$ HPDI } \\
\hline & & & Lower & Upper & & Lower & Upper \\
\hline \multirow[t]{3}{*}{ Male } & Resting & 0.41 & 0.41 & 0.42 & 0.41 & 0.40 & 0.42 \\
\hline & Foraging & 0.48 & 0.47 & 0.49 & 0.52 & 0.51 & 0.53 \\
\hline & Transit & 0.11 & 0.10 & 0.12 & 0.08 & 0.07 & 0.08 \\
\hline \multirow[t]{3}{*}{ Female } & Resting & 0.28 & 0.27 & 0.29 & 0.44 & 0.42 & 0.45 \\
\hline & Foraging & 0.65 & 0.64 & 0.66 & 0.50 & 0.48 & 0.52 \\
\hline & Transit & 0.07 & 0.06 & 0.08 & 0.06 & 0.05 & 0.07 \\
\hline
\end{tabular}

Note: Results are from two separate analyses using (1) both dive and location data and (2) location data only. HPDI is the highest posterior density interval.

and less time in the foraging state after 1 June (Table 2). Because females restrict distances from haul-out sites in early lactation (Thompson et al. 1994), this could be due to shorter foraging trips to return to suckle pups. Foraging is known to decrease in males during the breeding season (Boness et al. 2006), and we found males spent slightly more time resting and less time in the foraging state after 1 June.
Estimated movement characteristics were similar between the analysis utilizing both dive and location data and the analysis utilizing location data only. However, the estimated overall activity budgets were significantly different between the two analyses, with little difference in estimated activity budgets between the sexes when inference was based solely on location data (Table 1). When comparing these results with the known

Relative density
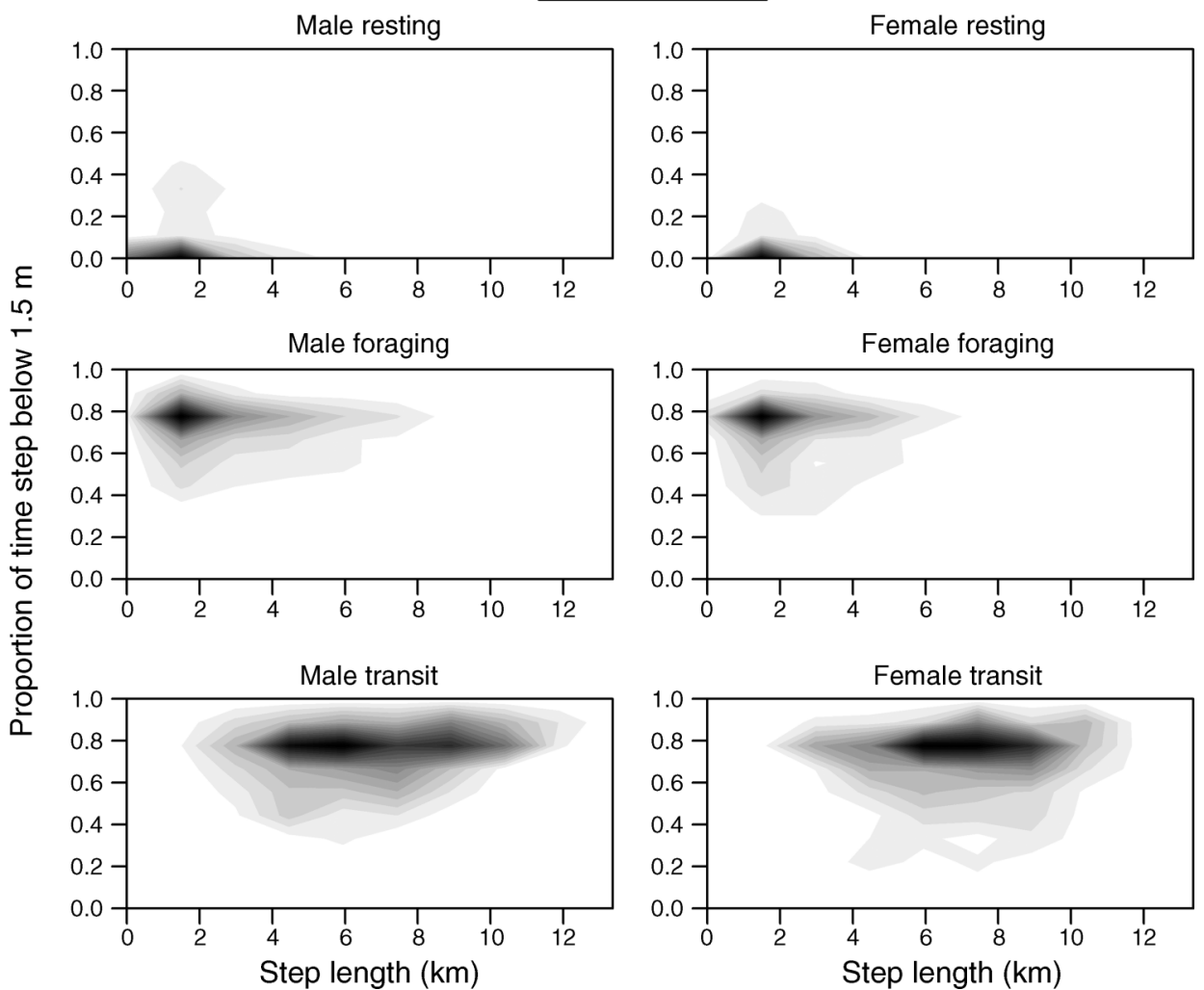

FIG. 5. Estimated bivariate densities of harbor seal step length and proportion of time step spent diving below $1.5 \mathrm{~m}$. Separate densities were estimated for males and females with three distinct movement behavior states (resting, foraging, and transit), where darker shades indicate higher relative densities. Results are from analysis using both dive and location data. Time steps are 120 minutes. 


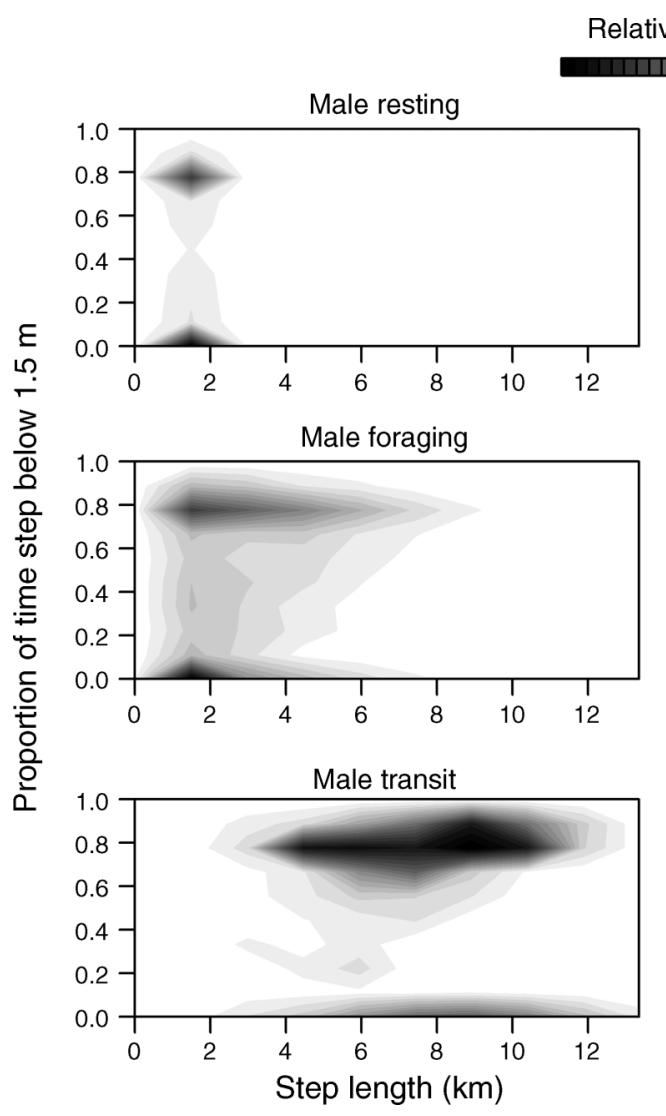

Relative density

तालालाप
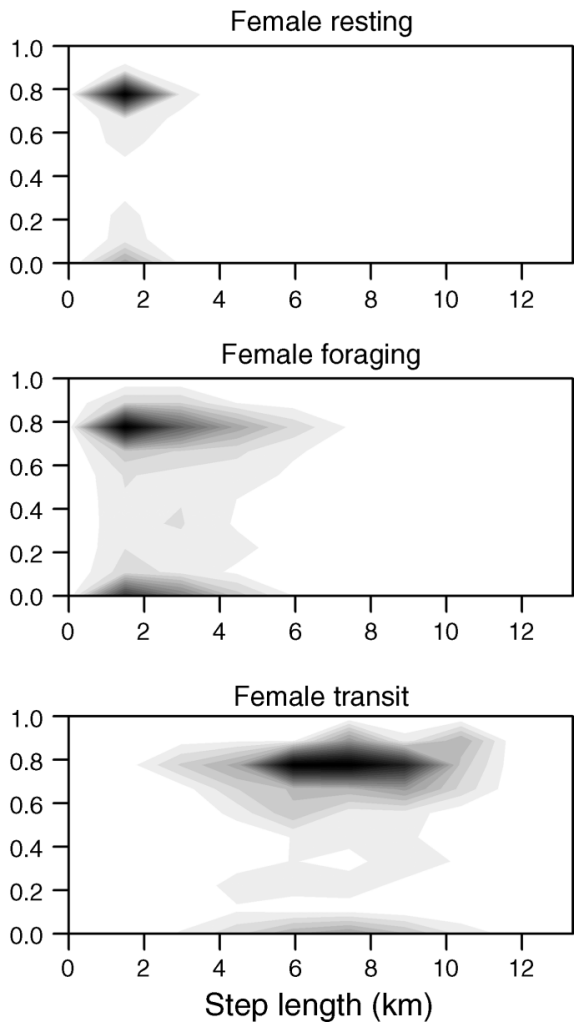

FIG. 6. Estimated bivariate densities of harbor seal step length and proportion of time step spent diving below $1.5 \mathrm{~m}$. Separate densities were estimated for males and females with three distinct movement behavior states (resting, foraging, and transit), where darker shades indicate higher relative densities. Results are from analysis using location data only. Time steps are 120 minutes.

proportion of time spent diving below $1.5 \mathrm{~m}$ for each time step (Fig. 6), it is evident that many intervals with a majority of respective time spent above or below $1.5 \mathrm{~m}$ were assigned to foraging/transit or resting. For example, $33 \%$ (95\% HPDI, 0.32-0.34) of time steps with $\omega_{n, t}>0.5$ were assigned to resting when based on horizontal trajectory alone, but only $1 \%$ (95\% HPDI, 0.01-0.02) of time steps with $\omega_{n, t}>0.5$ were assigned to resting when inferred from both horizontal trajectory and the dive activity data. Similarly, we found that $46 \%$
(95\% HPDI: $0.44-0.47)$ of time steps exhibiting a majority of non-diving activity (i.e., $\omega_{n, t}<0.5$ ) were assigned to foraging/transit when based on horizontal trajectory alone, but only $12 \%$ (95\% HPDI, 0.11-0.12) of these time steps were assigned to foraging/transit when using our integrated model. Because harbor seals are known to dive for foraging and transit (but not usually for resting), a large proportion of time steps were apparently mischaracterized when these behavior states were inferred from horizontal trajectory alone.

TABLE 2. Estimated proportion of 120 -minute time steps assigned to $Z=3$ movement behavior states (resting, foraging, and transit) for $N=17$ (10 male, 7 female) harbor seals in the United Kingdom.

\begin{tabular}{|c|c|c|c|c|c|c|c|}
\hline \multirow[b]{3}{*}{ Sex } & \multirow[b]{3}{*}{ Behavior state } & \multicolumn{3}{|c|}{ Pre-breeding } & \multicolumn{3}{|c|}{ Post-breeding } \\
\hline & & \multirow[b]{2}{*}{ Time allocation } & \multicolumn{2}{|c|}{ 95\% HPDI } & \multirow[b]{2}{*}{ Time allocation } & \multicolumn{2}{|c|}{ 95\% HPDI } \\
\hline & & & Lower & Upper & & Lower & Upper \\
\hline \multirow[t]{3}{*}{ Male } & Resting & 0.41 & 0.40 & 0.41 & 0.43 & 0.42 & 0.43 \\
\hline & Foraging & 0.49 & 0.48 & 0.50 & 0.45 & 0.44 & 0.47 \\
\hline & Transit & 0.11 & 0.10 & 0.11 & 0.12 & 0.11 & 0.13 \\
\hline \multirow[t]{3}{*}{ Female } & Resting & 0.28 & 0.27 & 0.29 & 0.28 & 0.27 & 0.29 \\
\hline & Foraging & 0.66 & 0.65 & 0.67 & 0.63 & 0.61 & 0.64 \\
\hline & Transit & 0.06 & 0.05 & 0.07 & 0.09 & 0.08 & 0.10 \\
\hline
\end{tabular}

Notes: Two time periods are compared: pre-breeding (prior to 1 June) and post-breeding (after 1 June). State assignments are based on both location and dive data for each time step. 
Although the movement and diving times associated with each movement behavior state are consistent with those expected for resting, foraging, and transit, biological interpretations of movement characteristics and activity budgets should be made with care. Because time steps were specified at 120-minute intervals, state switches within intervals were certainly possible. This may have introduced some state assignment error, additional uncertainty, or lack of model fit (see Appendix A). While only $10 \%$ of the diving proportions $\left(\omega_{n, t}\right)$ were between 0.4 and 0.6 , suggesting mid-interval switches between resting and foraging or transit were relatively rare, such switches may have somewhat respectively inflated or deflated the expected value of the resting or foraging/transit step length distribution. The potential for mid-interval switches between foraging and transit is more difficult to assess. The foraging state could also include underwater defending of territory for males, or resting underwater on the sea floor. To more accurately identify foraging, one would need to incorporate dive shape or information about feeding, such as stomach temperatures.

\section{Discussion}

Our ability to characterize movement and predict behavior states can be greatly improved by utilizing all potential sources of information. This includes data from multiple individuals, or data of an ancillary nature to movement. Although we have focused on combining animal location and biotelemetry data to investigate population-level activity budgets and associated movement characteristics, the proposed methodology could be used with any other sources of information that inform behavioral states. In the harbor seal example, GIS data could be utilized to more finely characterize resting (e.g., as resting at haul out and resting at sea) based on whether or not locations were on land. Similarly, bathymetry data could be used to inform different types of diving behavior (e.g., bearded seals forage on the ocean floor).

We have emphasized that the distribution of the model for ancillary biotelemetry data will necessarily depend on the nature of the data and the species of interest. In our harbor seal example and the hypothetical example illustrated in Fig. 1a (see The individual model), where the ancillary data were additional (categorical) spatial coordinates, conditioning the ancillary dive data on the latent states was a natural choice. However, when the ancillary data are measures of internal condition, it may be more sensible to condition the latent states on the ancillary data. Consider the hypothetical stomach temperature example described in Fig. 1b (see The individual model). If the animal is feeding, its stomach temperature is more likely to be lower, and if the animal is searching, perhaps its stomach temperature is more likely to be higher. In this case, it may be more natural to condition the states on stomach temperature $\left(\omega_{t}\right)$, e.g., $\left[z_{t} \mid \omega_{t}\right] \sim$ Categorical $\left(p_{\mathrm{R}, t}, p_{\mathrm{D}, t}, p_{\mathrm{S}, t}\right)$, where $p_{\mathrm{F}, t}$ and $p_{\mathrm{S}, t}$ are functions of $\omega_{t}$, and $p_{\mathrm{R}, t}=1-p_{\mathrm{D}, t}-p_{\mathrm{S}, t}$.

The potential application of the proposed methodology need not be limited to marine mammal species. For example, location transmitters equipped with accelerometers (e.g., Wilson et al. 2008) that record head movement could be used to distinguish behaviors of terrestrial predators as they stalk, attack, and consume prey. Head movement could also be used to help distinguish resting from grazing in herbivorous species.

As demonstrated by our harbor seal example, there is mounting evidence of the potential perils of inferring animal behavior based on trajectory alone. Regardless of the species of interest, there fortunately now exist many flexible analytical techniques for extracting more out of the increasing wealth of information afforded by recent advances in biologging technology. Advancing these analytical methods further remains a promising avenue for future research. At the individual level, these advances include the incorporation of continuous-time movement models (e.g., Blackwell 2003) or an unknown number of behavior states. At the population level, an overarching goal remains linking individual behavior with survival and reproduction to better understand population dynamics and predict population-level redistribution.

\section{ACKNOWLEDGMENTS}

We thank B. McConnell, D. Thompson, and P. Hammond for use of their data; B. McConnell, D. Thompson, P. Conn, D. Johnson, and J. London for helpful discussions; and S. Moss and M. Bivins for fieldwork. Data collection was supported by the Scottish Natural Heritage, Marine Scotland Science, Hampshire and Isle of Wight Wildlife Trust, and Chichester Harbour Conservancy. Some of this research was undertaken while B. McClintock was funded by the Engineering and Physical Science Research Council. D. Russell is funded by the U.K. Department of Energy and Climate Change as part of their Offshore Energy Strategic Environmental Assessment program. The findings and conclusions in the paper are those of the author(s) and do not necessarily represent the views of the National Marine Fisheries Service, NOAA. Any use of trade, product, or firm names does not imply an endorsement by the U.S. Government.

\section{Literature Cited}

Austin, D., W. D. Bowen, J. I. McMillan, and D. J. Boness. 2006. Stomach temperature telemetry reveals temporal patterns of foraging success in a free-ranging marine mammal. Journal of Animal Ecology 75:408-420.

Blackwell, P. G. 2003. Bayesian inference for Markov processes with diffusion and discrete components. Biometrika 90:613627.

Boness, D. J., W. D. Bowen, B. M. Buhleier, and G. J. Marshall. 2006. Mating tactics and mating system of an aquatic-mating pinniped: the harbor seal, Phoca vitulina. Behavioral Ecology and Sociobiology 61:119-130.

Breed, G. A., I. D. Jonsen, R. A. Myers, W. D. Bowen, and M. L. Leonard. 2009. Sex-specific, seasonal foraging tactics of adult grey seals (Halichoerus grypus) revealed by statespace analysis. Ecology 90:3209-3221.

Cooke, S. J., S. G. Hinch, M. Wikelski, R. D. Andrews, L. J. Kuchel, T. G. Wolcott, and P. J. Butler. 2004. Biotelemetry: a mechanistic approach to ecology. Trends in Ecology and Evolution 19:334-343. 
Cunningham, L., J. M. Baxter, I. L. Boyd, C. D. Duck, M. Lonergan, S. E. Moss, and B. J. McConnell. 2009. Harbour seal movements and haul-out patterns: implications for monitoring and management. Aquatic Conservation: Marine and Freshwater Ecosystems 19:398-407.

Eckert, S. A., J. E. Moore, D. C. Dunn, R. S. van Buiten, K. L. Eckert, and P. N. Halpin. 2008. Modeling loggerhead turtle movement in the Mediterranean: importance of body size and oceanography. Ecological Applications 18:290-308.

Frair, J. L., J. Fieberg, M. Hebblewhite, F. Cagnacci, N. J. DeCesare, and L. Pedrotti. 2010. Resolving issues of imprecise and habitat-biased locations in ecological analyses using GPS telemetry data. Philosophical Transactions of the Royal Society B 365:2187-2200.

Gaspar, P., J.-Y. Georges, S. Fossette, A. Lenoble, S. Ferraroli, and Y. Le Maho. 2006. Marine animal behaviour: neglecting ocean currents can lead us up the wrong track. Proceedings of the Royal Society B 273:2697-2702.

Hanks, E. M., M. B. Hooten, D. S. Johnson, and J. T. Sterling. 2011. Velocity-based movement modeling for individual and population level inference. PLoS ONE 6:e22795.

Higgs, M. D., and J. M. Ver Hoef. 2011. Discretized and aggregated: modeling dive depth of harbor seals from ordered categorical data with temporal autocorrelation. Biometrics. http://dx.doi.org/10.1111/j.1541-0420.2011.01710.x

Johnson, D. S., J. M. London, and C. E. Kuhn. 2011. Bayesian inference for animal space use and other movement metrics. Agricultural, Biological, and Environmental Statistics 16:357-370.

Jonsen, I. D., J. M. Flemming, and R. A. Myers. 2005. Robust state-space modeling of animal movement data. Ecology 86:2874-2880.

Jonsen, I. D., R. A. Myers, and J. M. Flemming. 2003. Metaanalysis of animal movement using state-space models. Ecology 84:3055-3063.

Jonsen, I. D., R. A. Myers, and M. C. James. 2006. Robust hierarchical state-space models reveal diel variation in travel rates of migrating leatherback turtles. Journal of Animal Ecology 75:1046-1057.

Jonsen, I. D., R. A. Myers, and M. C. James. 2007. Identifying leatherback turtle foraging behaviour from satellite telemetry using a switching state-space model. Marine Ecology Progress Series 337:255-264.

Langrock, R., R. King, J. Matthiopoulos, L. Thomas, D. Fortin, and J. M. Morales. 2012. Flexible and practical modeling of animal telemetry data: hidden Markov models and extensions. Ecology 93:2336-2342.

Le Boeuf, B. J., D. E. Crocker, D. P. Costa, S. B. Blackwell, P. M. Webb, and D. S. Houser. 2000. Foraging ecology of northern fur seals. Ecological Monographs 70:353-382.

McClintock, B. T., R. King, L. Thomas, J. Matthiopoulos, B. J. McConnell, and J. M. Morales. 2012. A general discrete-time modeling framework for animal movement using multi-state random walks. Ecological Monographs 82:335-349.

Morales, J. M., D. T. Haydon, J. Frair, K. E. Holsinger, and J. M. Fryxell. 2004. Extracting more out of relocation data: building movement models as mixtures of random walks. Ecology 85:2436-2445.

Patterson, T. A., L. Thomas, C. Wilcox, O. Ovaskainen, and J. Matthiopoulos. 2008. State-space models of individual animal movement. Trends in Ecology and Evolution 23:8794.

Russell, D. J. F., J. Matthiopoulos, and B. J. McConnell. 2011. SMRU seal telemetry quality control process. SCOS Briefing paper 11/17. Sea Mammal Research Unit, University of St. Andrews, Fife, UK.

Sharples, R. J., S. E. Moss, T. A. Patterson, and P. S. Hammond. 2012. Spatial variation in foraging behaviour of a marine top predator (Phoca vitulina) determined by a largescale satellite tagging program. PLoS ONE 7:e37216.

Sparling, C. E., M. A. Fedak, and D. Thompson. 2007. Eat now, pay later? Evidence of deferred food-processing costs in diving seals. Biology Letters 3:95-99.

Thompson, P. M., D. Miller, R. Cooper, and P. S. Hammond. 1994. Changes in the distribution and activity of female harbor seals during the breeding season: implications for their lactation strategy and mating patterns. Journal of Animal Ecology 63:24-30.

Wilson, R. P., E. L. C. Shepard, and N. Liebsch. 2008. Prying into the intimate details of animal lives: use of a daily diary on animals. Endangered Species Research 4:123-137.

\section{Supplemental Material}

\section{Appendix A}

Prior specifications, prior sensitivity, and goodness of fit for the harbor seal example (Ecological Archives E094-072-A1).

\section{Appendix B}

Markov chain Monte Carlo algorithm (Ecological Archives E094-072-A2).

\section{Appendix C}

Posterior summaries for the harbor seal example (Ecological Archives E094-072-A3).

\section{Supplement}

Source code and example data for implementing the Markov chain Monte Carlo algorithm (Ecological Archives E094-072-S1). 